\title{
Criminologie
}

\section{Les fraudes fiscales collectives durables : étude d'un réseau de facturation de complaisance}

\section{Julie Paquin}

Volume 37, numéro 2, automne 2004

Délinquance et réussite

URI : https://id.erudit.org/iderudit/010708ar

DOI : https://doi.org/10.7202/010708ar

Aller au sommaire du numéro

\section{Éditeur(s)}

Les Presses de l'Université de Montréal

ISSN

0316-0041 (imprimé)

1492-1367 (numérique)

Découvrir la revue

Citer cet article

Paquin, J. (2004). Les fraudes fiscales collectives durables : étude d'un réseau de facturation de complaisance. Criminologie, 37(2), 151-175.

https://doi.org/10.7202/010708ar
Résumé de l'article

La forme que prend la fraude fiscale complexe à l'étude est celle d'un marché de factures de complaisance qui a desservi 350 compagnies appartenant à l'industrie montréalaise du vêtement pendant une décennie. Cette fraude est connue sous le nom de l'affaire "Ventex ». L'examen du cas est principalement basé sur les informations tirées des transcriptions judiciaires des procès qui ont découlé de la découverte du scandale. Nous avons également réalisé des entrevues avec des témoins directs et indirects de l'affaire pour compléter les renseignements à notre disposition.

En analysant l'affaire "Ventex ", nous examinons trois thématiques. La thématique du succès nous amène à étudier les conditions qui ont assuré la viabilité et la pérennité de ce marché spécifique de factures d'accommodation. La thématique de son impunité est justifiée en partie par la tolérance de régulateurs habituels de performance économique des compagnies. Enfin, la thématique du contrôle judiciaire est abordée de façon à expliquer pourquoi l'affaire "Ventex " a donné lieu à des poursuites criminelles, alors que les tribunaux criminels sont rarement sollicités pour sanctionner la délinquance d'affaires. En reconstituant la dynamique de cette fraude fiscale complexe, nos résultats mettent en rapport différents aspects d'un phénomène criminel généralement abordés séparément dans la littérature consacrée à la fraude fiscale. 


\title{
Les fraudes fiscales collectives durables: étude d'un réseau de facturation de complaisance ${ }^{1}$
}

\author{
Julie Paquin \\ M.Sc. en criminologie \\ Université de Montréal
}

\begin{abstract}
RÉSUMÉ - La forme que prend la fraude fiscale complexe à l'étude est celle d'un marché de factures de complaisance qui a desservi 350 compagnies appartenant à l'industrie montréalaise du vêtement pendant une décennie. Cette fraude est connue sous le nom de l'affaire "Ventex». L'examen du cas est principalement basé sur les informations tirées des transcriptions judiciaires des procès qui ont découlé de la découverte du scandale. Nous avons également réalisé des entrevues avec des témoins directs et indirects de l'affaire pour compléter les renseignements à notre disposition.

En analysant l'affaire "Ventex», nous examinons trois thématiques. La thématique du succès nous amène à étudier les conditions qui ont assuré la viabilité et la pérennité de ce marché spécifique de factures d'accommodation. La thématique de son impunité est justifiée en partie par la tolérance de régulateurs habituels de performance économique des compagnies. Enfin, la thématique du contrôle judiciaire est abordée de façon à expliquer pourquoi l'affaire «Ventex» a donné lieu à des poursuites criminelles, alors que les tribunaux criminels sont rarement sollicités pour sanctionner la délinquance d'affaires. En reconstituant la dynamique de cette fraude fiscale complexe, nos résultats mettent en rapport différents aspects d'un phénomène criminel généralement abordés séparément dans la littérature consacrée à la fraude fiscale.
\end{abstract}

ABSTRACT - Using a case study, we intend to explain the scope and the life-length of an accommodation invoices' market. We chose to study Ventex's case, which is a tax evasion scheme related to Montreal's garment industry that attracted 350 companies during ten years. Our principal source of information are the judicial transcripts of criminal lawsuits and civil actions. We also gathered testimonies of individuals related directly or indirectly to the case.

1. L'auteure tient à remercier M. Pierre Tremblay, professeur à l'École de criminologie de l'Université de Montréal, pour ses encouragements et ses suggestions lors des versions préliminaires de cet article. 
Three theses are examined in this paper. First, the success thesis leads us to study the conditions which ensured the viability and the life-length of the accommodation invoices' market. Second, the bank's employees passive and active collusion with the accommodator is approached in the impunity thesis we develop. At last, the legal outcome of the tax evasion scheme's disclosure, exceptional since criminal prosecution is scarce in white-collar crime cases, is studied in the judicial control thesis. By replicating in part the methodology used in contextual analysis, we aim to expose the dynamics of a complex fraud and to put together various aspects of a criminal phenomenon generally approached separately in the literature devoted to tax evasion.

\section{Introduction}

Les études consacrées à la fraude fiscale se sont majoritairement penchées sur les pratiques dévoyées des particuliers, plutôt que sur celles des compagnies. Elles s'intéressent au contribuable et à son comportement à travers des questionnaires de délinquance fiscale autorévélée (Stalans $e t$ al., 1987; Varma et Doob, 1998), des analyses empiriques et économétriques basées sur la consultation des dossiers fiscaux des particuliers (Klepper et Nagin, 1987; Cowell, 1991; Elffers, 1991) et des recherches expérimentales sur les délits fiscaux (Pyle, 1989; Thurman, 1989). Les auteurs recensés examinent la décision de frauder ou de produire une déclaration de revenus honnête en fonction des processus de dissuasion et de sanctionnement qui y sont rattachés. Dans une moindre mesure, les opportunités qui peuvent être exploitées par les fraudeurs sont considérées (Cowell, 1991; Elffers, 1991; Friedrichs, 1996). En outre, les contrôles sociaux formels et informels et leur impact sur les pratiques fiscales délinquantes sont étudiés à travers la perception du risque de détection de la fraude (Klepper et Nagin, 1989; Varma et Doob, 1998), la certitude d'être pénalisé (Shover et Bryant, 1993) et la distance temporelle de la sanction (Stalans et al., 1987; Varma et Doob, 1998). Finalement, certains travaux abordent les facteurs économiques et politiques comme le taux de taxation et d'imposition (Clotfelter, $1983^{2}$; Nagin et Klepper, 1987), le contexte économique et la satisfaction envers les gouvernements et les services offerts à la population (Alms, 1996).

Le traitement quasi exclusif des travaux empiriques consacré à la fraude fiscale des particuliers doit être mis en contexte à l'aide des données du ministère du Revenu du Québec: sur les 1547 millions de

2. Tiré de Cowell, 1991. 
dollars récupérés en taxes et en impôts éludés, près des trois quarts de la somme l'ont été auprès d'entreprises $(73 \%)$ et un peu plus du quart, auprès de particuliers $(27 \%)$. Cette situation est surprenante considérant que les individus contribuent pour $70 \%$ des revenus au fédéral et les compagnies, $19 \%$ (Bernard et al., 1995). Ces chiffres soulignent le manque d'études traitant de la fraude fiscale des corporations.

Dans cet article, nous procédons par voie d'étude de cas pour analyser la dynamique d'une fraude fiscale collective impliquant un grand nombre de manufacturiers de vêtements pour dames établis à Montréal. Nous nous inspirons d'un courant de recherche dont la vertu particulière est de contextualiser la délinquance d'affaires en la resituant dans le cadre des contingences qui caractérisent leur secteur spécifique d'activité économique : l'industrie automobile (Leonard et Weber, 1970; Faberman, 1975), l'industrie des déchets (Reuter, 1993), l'industrie des spiritueux (Denzin, 1977) ou celui de l'industrie de l'équipement électrique industriel (Baker et Faulkner, 1993). Ces analyses identifient les conditions sociales et économiques dans lesquelles émergent des transactions illicites, les entreprises et les entrepreneurs impliqués, ainsi que les pouvoirs de législation et d'application de la loi.

Certaines recherches portant sur la criminalité d'organisations ou d'industries incluent, entre autres délits, des fraudes fiscales (par exemple Clinard, 1979), mais peu d'entre elles s'attardent uniquement à ce sujet et l'analysent en profondeur. Clinard explique la difficulté d'accéder à l'information sur les fraudes fiscales des compagnies par le traitement qu'en fait le Service du revenu américain. Elles font la plupart du temps l'objet de procédures administratives internes et engendrent des poursuites légales uniquement lorsqu'elles sont portées en appel devant un tribunal fédéral.

Nous avons identifié une étude qui, sans s'attarder spécifiquement à un secteur de l'économie, s'apparente à notre recherche par la forme de fraude fiscale décrite. Dans son article «Tax evasion by proxy: the gray market in welfare capitalism», Barnett (1984) traite de la fraude fiscale comme moyen de camoufler un marché "gris» de l'emploi en Suède. L'auteur démontre dans ce travail comment ce marché parallèle de maind'œuvre s'est développé comme solution aux problèmes engendrés par l'encadrement légal de l'embauche et par le fardeau fiscal. Il trace un portrait de la fraude fiscale beaucoup plus complexe que ne l'était celui des délits fiscaux des particuliers. Les motivations sont autres: les employeurs ultimes visent d'abord l'efficacité de leur entreprise plutôt 
que de retirer directement des gains financiers. En outre, le modus operandi est plus élaboré et il implique un plus grand nombre de participants. Cet exemple de fraude fiscale complexe, qui sous-tend le marché parallèle de main-d'œuvre, dépeint l'imbrication de plusieurs formes de délits, l'association nécessaire entre firmes et la variété des motivations des acteurs en fonction de leurs objectifs corporatifs.

La forme de fraude fiscale corporative que nous avons choisi d'étudier, soit la vente et l'achat de factures de complaisance, a la particularité, comme le marché gris décrit par Barnett, de donner lieu à un marché parasite de transactions. Ce marché, qui s'est développé au sein de l'industrie montréalaise du vêtement pour dames dans les années 1960 et 1970, est analysé à travers l'affaire «Ventex» (du nom de la compagnie qui offrait ces services). Les compagnies appartenant à ce secteur de l'économie sont pour la plupart de petite ou de moyenne taille et sont caractérisées par une structure interne faiblement hiérarchisée. On retrouve en effet, à la tête de ces compagnies, un propriétaire ou un groupe d'actionnaires limité à trois ou quatre individus qui s'occupent directement de la gestion de l'entreprise et de l'exécution des tâches administratives courantes. Ces entreprises évoluent dans un marché hautement compétitif au sein duquel plusieurs échanges légitimes de biens (tissus et vêtements) et de services (confection de vêtements) ont cours entre fournisseurs et acheteurs. La fraude fiscale à l'étude repose sur des transactions fictives des mêmes biens et services. Contrairement à ce qui est présenté dans la littérature empirique sur la fraude fiscale des particuliers, les pratiques de factures de complaisance ne peuvent être réalisées solitairement. Elles exigent la participation d'un grand nombre de «clients», tiers extérieurs à la compagnie, et doivent s'intégrer au circuit des transactions légitimes pour mieux se dissimuler. Nous avons donc affaire à un délit fiscal basé sur la coopération de deux ou plusieurs sociétés dont les alliances ont la durée d'une transaction. Cette forme de fraude fiscale a pour objectif de diminuer le fardeau fiscal des entreprises et les coûts de production des biens manufacturés. La conjugaison de l'opportunité générée par la structure de l'industrie et de l'utilité de ce délit fiscal pour la compétitivité de l'entreprise expliquent sa prévalence et sa longévité.

L'analyse de l'affaire «Ventex» vise, d'une part, à identifier les conditions d'émergence et de viabilité d'une fraude fiscale au sein d'un marché légal de production de biens. Outre le gain tiré d'une activité économique illégale, nous avons voulu, comme l'a fait Barnett (1984), 
comprendre les impératifs économiques propres à un tel marché, les motivations des producteurs de tissus et de vêtements, ainsi que des régulateurs habituels de performance économique comme les comptables et les banquiers, à s'impliquer dans une telle activité. Nous nous questionnons, d'autre part, sur la forme que prend le contrôle judiciaire dans une affaire comme celle de "Ventex»: comment expliquer les poursuites criminelles alors que les tribunaux criminels sont rarement sollicités pour sanctionner la délinquance d'affaires? En procédant à cette étude de cas et en reconstituant la dynamique de cette fraude fiscale complexe, nos résultats mettent en rapport les différents aspects d'un phénomène criminel, alors qu'ils sont généralement abordés séparément dans la littérature consacrée à la fraude fiscale. L'intégration dans une analyse du modus operandi et de la réaction judiciaire a l'avantage d'augmenter sa valeur explicative par l'étude de leur influence mutuelle.

\section{Données et stratégie d'analyse}

Pour étudier l'affaire «Ventex», qui consiste en un schème de facturation fictive dans l'industrie du vêtement à Montréal, nous avons reproduit en partie la méthode d'analyse contextuelle. Ce cas a l'avantage de nous donner accès à un large éventail de données et de nous permettre d'examiner la forme que prennent les pratiques de facturation fictive sous différents points de vue. L'examen d'un cas mis au jour en 1976 se justifie en outre par la lenteur des procédures judiciaires. En effet, les fraudes complexes comme celles entourant les activités de la société Ventex entraînent un nombre important de poursuites qui peuvent s'échelonner sur plusieurs années. L'examen de l'ensemble des poursuites criminelles et civiles intentées, de l'identité des parties impliquées, mais aussi de l'ordre des démarches judiciaires entreprises, témoignent des motivations réelles des participants. Leur intégration enrichit l'étude et améliore la compréhension de la fraude, notamment par la preuve présentée à la cour qui est rendue disponible. Dans le cas de l'affaire «Ventex», les deux dernières poursuites se sont conclues en 1994 et en 1996. La rareté et l'éloquence des démarches judiciaires dans l'investigation de la fraude justifient, à notre sens, le choix de cette affaire, malgré la distance temporelle qui nous sépare de sa découverte.

Notre principale source d'information est la transcription judiciaire des procès criminels et civils qui ont été entrepris à la suite du déclenchement de l'enquête sur l'affaire, bonifiée par des informations 
détaillées et diversifiées contenues dans les preuves présentées en cour ${ }^{3}$. Le fait que des poursuites pénales aient été entreprises à la suite du dévoilement de l'affaire rend notre cas atypique par rapport à la criminalité des corporations, qui sont rarement l'objet de telles mesures.

Nous avons aussi dépouillé des articles de journaux consacrés à cette affaire. Ils constituent une source privilégiée d'information dans la mesure où ils nous permettent de reconstituer la trame des événements, lorsque les rapports officiels d'enquête ont été détruits, et parce qu'ils consignent un ensemble d'informations qui ne sont pas mentionnées dans les transcriptions des procès, mais qui sont fort utiles lorsqu'il s'agit de reconstituer rétrospectivement l'historique de cette affaire. La majorité de ces articles proviennent du quotidien montréalais The Gazette. Nous avons également consulté une dizaine d'articles préservés dans les archives de ce quotidien ${ }^{4}$.

En ce qui a trait aux autres sources d'information écrites, nous nous sommes tournée principalement vers des documents rédigés par différents ministères ou organismes gouvernementaux ${ }^{5}$. Ces renseignements nous permettent de comprendre la situation économique et politique de ce secteur manufacturier, afin de remettre dans son contexte la pratique de facturation fictive. Ils ne remplacent toutefois pas une littérature scientifique sur les pratiques illicites dans ce domaine de l'économie, pratiquement inexistante dans les champs de la criminologie, de la sociologie et de l'économie.

Nous avons également voulu recueillir les témoignages d'individus reliés de près ou de loin à notre sujet. Dans cette optique, nous avons dans un premier temps approché les organismes de contrôle social liés à la fraude fiscale. Des entretiens ont été menés avec un agent du ministère du Revenu fédéral chargé à l'époque de l'enquête Shildan/Ventex. En outre, au Québec, le ministère du Revenu provincial est particuliè-

3. Nous avons, par exemple, la liste des comptes recevables de Ventex sur plusieurs mois, ainsi que l'analyse qu'en a fait un expert-comptable, le rapport d'application de crédit de Shildan rédigé par la Banque Toronto Dominion, ainsi qu'une série de copies des chèques et des factures fictives transigées entre la compagnie accommodatrice et ses clients, identifiés dans une liste détaillée.

4. M. William Marsden, rédacteur en chef de The Gazette, nous a aimablement donné accès aux archives du journal.

5. À titre d'exemple: «Le budget 1998-99 du ministère des Finances du Québec », des données descriptives sur le vêtement et le textile compilées par Statistique Canada, ainsi qu'un document sur le secteur de la mode publié par le ministère de l'Industrie et du Commerce du Québec. 
rement actif dans la lutte à la facturation fictive et au travail au noir dans l'industrie du vêtement. Nous avons donc rencontré les chargés de projet affectés à cette opération, qui nous ont expliqué le fonctionnement de la facturation fictive ainsi que des mesures entreprises pour lutter contre cette forme de fraude fiscale. Dans un deuxième temps, nous avons approché le journaliste William Marsden du quotidien The Gazette, qui a publié régulièrement et de façon détaillée des articles sur le déroulement des enquêtes, ainsi que sur les procès rattachés à ce que la presse de l'époque qualifiait de «the great garment trade tax evasion scam».

\section{L'étendue du marché des factures de complaisance}

Le 8 juin 1976, le quotidien The Gazette révèle l'existence d'une fraude fiscale organisée dans l'industrie du vêtement pour dames à Montréal. Un manufacturier de textiles du nom de Leonard Cohen aurait fourni sur une période de 10 ans des factures de complaisance à près de 350 manufacturiers de vêtements, leur permettant ainsi d'éluder 64 millions de dollars d'impôts.

L'échange de factures d'accommodation a pour objectif de frauder le gouvernement en permettant à l'acheteur d'augmenter artificiellement ses coûts de production et, conséquemment, de diminuer ses bénéfices imposables. L'argent comptant ainsi généré peut être utilisé à titre personnel par l'acheteur de factures. Il est exempt d'imposition personnelle puisqu'il n'est pas déclaré. Le vendeur jouit pour sa part de la commission retirée de la vente de telles factures et peut utiliser le gonflement de ses comptes à recevoir comme garantie pour obtenir des avantages pécuniaires des organismes financiers comme les banques. Dans le cas à l'étude, le vendeur diminue le volume important de ventes générées par l'activité illégale par des achats fictifs de biens ou de services afin de ne pas gonfler exagérément son revenu imposable.

La transaction est caractérisée par l'absence d'échange de marchandises entre le vendeur (accommodateur) et l'acheteur (accommodé). L'acheteur détermine le montant de la facture et le type de marchandise qui doit y apparaître de façon à la rendre crédible aux yeux des vérificateurs de l'impôt, alors que le vendeur fixe le pourcentage de commission qu'il retire de la transaction. Afin d'illustrer une transaction type, admettons une commission de $5 \%$ appliquée à une facture de 10000 \$. Dans un premier temps, en réaction à la commande d'un 
client, l'accommodateur rédige la facture qui répond aux spécifications de l'acheteur et la lui livre, le plus souvent en main propre. Cette facture représente un faux achat pour le client. Lorsqu'il est prêt à honorer la facture, un représentant de la compagnie accommodatrice se procure 10000 \$ comptant à travers une entreprise de façade et remet la somme de 9500 \$ au client, après avoir retranché la commission $(500 \$)$. Il reçoit en retour un chèque de $10000 \$$, à titre de paiement pour l'achat en question, qui est généralement encaissé le jour même.

Leonard Cohen est l'actionnaire et unique propriétaire des deux compagnies placées au centre de la vente de factures dans le marché: Ventex et Shildan. En 1966, il a enregistré la raison sociale Ventex avec laquelle il a réalisé la majorité de ses transactions réelles et fictives. En 1974, Cohen incorpore une compagnie du nom de Shildan Knitting Mills Limited qui rachète les avoirs et les dettes de Ventex. Cette dernière devient, en août de cette même année, théoriquement inactive, mais Cohen continue d'utiliser le nom Ventex après l'incorporation de Shildan pour son commerce d'accommodation ${ }^{6}$. Shildan pour sa part réalise des affaires légales dans le tissage, possède une marge de crédit importante et produit annuellement une déclaration fiscale. Cohen a pour bras droit Harry Hartman qui est le directeur des ventes de ses compagnies. C'est lui qui s'occupe de la gestion quotidienne des factures d'accommodation auprès des banques et des clients. Le comptable de Shildan, un ami d'enfance de Cohen, se nomme Sam Karpman. Il est aussi de mèche dans les transactions de factures de complaisance. Enfin, chacun des quatre vendeurs de Shildan est attitré à un client auprès duquel il assure la vente de marchandises et de factures de complaisance.

\section{Les conditions requises pour un marché viable de factures de complaisance}

La taille du marché des factures de complaisance peut être estimée de différentes façons mais dans tous les cas, elle témoigne de la popularité des services rendus par Leonard Cohen au sein de l'industrie du vêtement. Selon le nombre de compagnies qui ont été amenées à faire des

6. Les informations à notre disposition sont tirées des transcriptions judiciaires des procès portant principalement sur les activités de Cohen de 1974 à 1976, au moment où Shildan était incorporée. Afin de simplifier le texte, nous utiliserons indifféremment le nom de cette compagnie, peu importe l'époque dont il sera question. 
déclarations volontaires, à la suite du dévoilement de l'affaire dans les médias, une première évaluation s'établit à $27 \%$ de l'industrie du vêtement montréalaise ${ }^{7}$. Toutefois, cette estimation nous semble minimale, notamment parce qu'elle ne prend pas en considération la taille des entreprises impliquées dans le marché de factures d'accommodation. En effet, l'étude des registres comptables de la compagnie Shildan révèle qu'en valeur monétaire, les ventes mensuelles de factures d'accommodation par compagnie sont au moins deux fois plus élevées que les ventes réelles. Le montant de l'achat doit être, d'une part, proportionnel au volume de production et, d'autre part, inférieur au volume des ventes réalisées pour ne pas générer des pertes dans les bilans financiers de la compagnie. Par conséquent, si les achats de factures d'accommodation chez Shildan sont en moyenne d'un montant plus élevé que les achats de tissus, les accommodés doivent logiquement être des compagnies dont le volume de production est supérieur à celui des vrais acheteurs de marchandise. Force est de conclure que la part de marché de factures d'accommodation de Cohen dépasse vraisemblablement $27 \%$ de l'industrie montréalaise. Un autre angle d'approche a été proposé par l'enquêteur en chef de Revenu Canada qui a travaillé sur l'affaire Shildan. Il a suggéré que Cohen songe spécifiquement au secteur du vêtement pour dames lorsqu'il dit accommoder la plus grande partie de l'industrie. Dans cette éventualité, la proportion des manufacturiers accommodés grimpe à près de $60 \%{ }^{8}$.

\section{Finalités des factures de complaisance}

Un marché se développe normalement en fonction de l'utilité du produit offert, comme Barnett (1984) l'a décrit au sujet de la fraude fiscale mise en œuvre pour contourner les normes du travail en Suède. Dans le cas présent, l'achat de factures d'accommodation a permis de soutenir d'autres pratiques illégales directement liées aux activités manufacturières de l'industrie du vêtement.

7. Statistique Canada évalue le nombre d'établissements à 1287 en 1975.

8. Les données de Statistique Canada pour l'année 1972 indiquent l'existence de 603 compagnies produisant des vêtements pour dames dont 350 compagnies accommodées, soit une proportion de $58 \%$. Industrie manufacturière du Canada: Niveau infraprovincial (1972). Catalogue annuel 31-209.N.B. Les données pour 1975 étant agrégées, elles ne nous permettent pas d'établir la somme des entreprises en fonction pour cette année. 
Le travail au noir

La première activité illicite soutenue par l'argent retiré de la facturation de complaisance est l'emploi de travailleurs au noir. L'industrie de la manufacture de vêtements est en effet une industrie à haute intensité de main-d'œuvre, c'est-à-dire que la production du bien est basée sur des ressources humaines plutôt que sur de la machinerie. Pour diminuer les coûts de production, les manufacturiers ont donc recours à des employés dont le travail n'est pas déclaré au gouvernement. Cette pratique leur permet de diminuer le taux horaire par rapport à ce qui est normalement imposé par les syndicats et elle les soulage des charges sociales et fiscales auxquelles ils sont soumis'. À cela s'ajoute que les manufacturiers font également face à des contraintes syndicales, notamment aux chapitres du surtemps et du recours aux travailleurs à domicile ou aux sous-traitants qui leur permettent de réagir rapidement à une demande volatile ${ }^{10}$.

\section{Les pots-de-vin}

La seconde pratique illégale a trait à la mise en marché et à la distribution des biens produits. Une façon de soutenir les ventes est de distribuer des pots-de-vin aux acheteurs des grands magasins ${ }^{11}$. La disponibilité d'argent comptant facilite la corruption d'acheteurs de commerces de détail auprès desquels certains manufacturiers tentent d'assurer l'écoulement de leur marchandise. Le phénomène observé chez les manufacturiers de vêtements est une des formes de corruption «privée» décrite par Ackerman (1978). Dans la même veine, Clinard et Yeager (1980) observent que les entreprises qui évoluent dans des marchés hautement compétitifs sont davantage susceptibles de s'impliquer dans ce type de délit. Les acheteurs de firmes de grande ou de moyenne taille sont souvent soudoyés par les cadres de leurs fournisseurs du secteur manufacturier et ce, à tous les niveaux de production (Clinard, 1979; Clinard et Yeager, 1980).

9. Sur les charges sociales et fiscales auxquelles se soustraient les manufacturiers du vêtement qui paient leurs employés «sous la table», voir le Discours sur le budget 1998-1999 du ministère des Finances du Québec (Favoriser l'intégrité fiscale, 1998 : 34).

10. Information tirée d'un mémoire adressé à madame Monique Bégin, avril 1977 : document tiré du dossier «Crabe» de la CECO.

11. Cette information est confirmée par certains manufacturiers lors de témoignages présentés devant des comités paritaires. 


\section{L'intégration économique du fournisseur de factures de complaisance}

Il ne suffit pas qu'il y ait une demande de factures de complaisance. Encore faut-il que l'offre de ces factures présente un certain nombre de caractéristiques propres à rassurer les acheteurs. Deux éléments doivent être soulignés : d'une part, Leonard Cohen était un véritable entrepreneur dans l'industrie du vêtement et, d'autre part, l'utilisation qu'il faisait des compagnies satellites qu'il avait mis sur pied permettait de simuler une apparente conformité fiscale.

L'intégration du fournisseur dans l'industrie du vêtement montréalaise est un élément clé dans l'explication de l'ampleur et de la longévité du stratagème frauduleux. La position de Cohen à la tête d'une bonneterie a généré d'abord des opportunités de dissimulation, puisqu'elle lui a permis de fondre les indices de fraude dans les pratiques commerciales légales. D'une part, il disposait des installations matérielles pour justifier au gouvernement les ventes de textile apparaissant sur les factures de complaisance vendues, ce qui n'aurait pas été le cas d'une compagnie fantôme. D'autre part, le textile était un bien dont avaient besoin les accommodés dans la production de vêtements. Les factures de complaisance étaient par conséquent parfaitement plausibles à première vue.

Onze entreprises satellites appartenant à Cohen ont été utilisées pour convertir les chèques des clients accommodés en liquide. Parmi celles-ci, deux sociétés de façade, Debco et Riegel, ne produisaient pas de déclaration fiscale. Le risque aurait été très grand de faire une déclaration d'impôt, puisque si une de ces compagnies avait été l'objet de vérifications par le ministère du Revenu, Hartman, le bras droit de Cohen, aurait dû justifier les retraits substantiels du compte à son nom propre.

Le second avantage lié à l'utilisation d'entreprises fictives est de permettre à Cohen de simuler des achats de Shildan chez Debco et Riegel afin de diminuer les revenus engendrés par la vente de factures d'accommodation. En utilisant des compagnies lui appartenant pour simuler des ventes à Shildan, Cohen pouvait ajuster les montants de ces achats fictifs en fonction des bénéfices qui devaient être présentés à la Banque Toronto Dominion pour assurer le maintien de sa marge de crédit. Le volume des ventes de factures d'accommodation étant tenu constant, moins les achats faits au sein des compagnies étaient élevés, plus Shildan générait des bénéfices. Le potentiel de réajustement entre les compagnies était infini et d'autant plus facile à effectuer que la 
banque prêteuse n'avait pas accès aux comptes bancaires des entreprises de façade, qui étaient situés dans d'autres institutions financières.

Cohen avait compris que ses entreprises satellites avaient peu de chances de faire l'objet de vérifications par la banque et qu'il pouvait exploiter cet avantage pour la réalisation et le déguisement de ses ventes de factures de complaisance. En ouvrant des comptes bancaires à différents endroits, Cohen a parfaitement su utiliser ces ressources nécessaires au bon fonctionnement de ses activités, puisqu'elles lui permettaient de morceler l'information à laquelle chaque institution financière avait accès.

\section{Des vertus du conformisme fiscal}

La principale raison pour laquelle le ministère du Revenu n'a jamais procédé à un examen approfondi de ses déclarations fiscales est que Cohen déclarait au fisc ses ventes d'accommodation ainsi que des bénéfices proportionnels à ses ventes annuelles, incluant les ventes fictives. Ce faisant, il se protégeait lui-même en même temps que ses clients ${ }^{12}$. Ainsi, si les enquêteurs du fisc s'intéressaient aux activités d'une entreprise acheteuse, les achats fictifs effectués chez Shildan, consignés dans la comptabilité, concordaient avec les ventes à cette compagnie apparaissant dans la liste de comptes à recevoir de Shildan ${ }^{13}$. En outre, si Shildan n'avait déclaré aucun bénéfice sur les ventes fictives, qui représentaient entre $70 \%$ et $75 \%$ de l'ensemble de ses ventes, ses activités auraient paru suspectes aux yeux du ministère du Revenu. Pour résoudre ce problème, il déclarait une proportion de la commission des ventes de factures d'accommodation à titre de bénéfices. La véracité des transactions de Cohen n'a donc jamais été remise en question parce qu'il a accepté, dès le départ, de limiter les revenus tirés de ses activités d'accommodation.

En bref, l'achat de factures d'accommodation représente une solution parfaitement adaptée aux besoins particuliers de l'industrie du vêtement,

12. Cohen témoigne lui-même du fait qu'il payait ses impôts sur les bénéfices tirés de ses transactions de factures d'accommodation (R. c. Cohen, t.j. [27/04/81], t. de L. Cohen: 179-180) et que c'est la raison pour laquelle son commerce d'accommodation a duré si longtemps (D.F. c. Shildan, t.j. [22/09/76], t. de L. Cohen : 148).

13. Le livre de comptes à payer d'une entreprise est composé de la liste des noms des compagnies auprès desquelles elle a fait des achats et des montants des achats. À l'inverse, le livre de comptes à recevoir est composé de la liste des noms des compagnies auprès desquelles elle a fait des ventes et des montants de ces ventes. 
puisqu'il permet à la fois de générer des sommes d'argent comptant non déclaré et aussi de créer des dépenses fictives équivalentes qui peuvent être déduites du revenu imposable et qui permettent, ironiquement, d'établir un portrait plus juste du bénéfice réel de l'entreprise aux yeux du gouvernement. Ce «réajustement» des états financiers de l'entreprise a en outre le mérite, pour les accommodés, d'éviter d'attirer les soupçons du ministère du Revenu et un contrôle éventuel de leur comptabilité.

En dépit de la "perfection» de cette fraude fiscale, le système mis en place efficacement par Cohen n'a pas résisté à la récession qui a frappé l'industrie du vêtement pour dames au milieu des années 1970. Lorsque Cohen a réalisé qu'il faisait face à d'importantes pertes financières, il a tenté d'intensifier ses ventes de factures d'accommodation. Cette tentative s'est soldée par un échec pour deux raisons. Premièrement, les acheteurs potentiels n'avaient pas besoin de davantage d'argent comptant au noir. Deuxièmement, les bénéfices que retirait Cohen de son commerce de factures de complaisance constituaient un revenu d'appoint et non pas une source de revenu principal. Malgré la nature organisée et systématique du marché de factures fictives mis sur pied, les revenus de cette activité demeuraient marginaux comparativement aux revenus légaux brassés par les protagonistes impliqués. C’est précisément lorsque Cohen a voulu que ses ventes de factures de complaisance lui procurent une source importante de revenus pour renflouer ses pertes de revenus dans la vente de produits textiles que la combine s'est effondrée. Ses alliés se sont retirés du jeu et les tensions internes de sa compagnie ont eu pour effet de mécontenter certains de ses employés qui se sont empressés de le dénoncer aux banques et aux corps policiers.

\section{Les alliés incertains}

Le succès d'un délit de l'ampleur du marché de facturation de complaisance étudié ne peut s'expliquer entièrement par l'organisation méticuleuse de la mécanique frauduleuse. L'intégration de Cohen dans le secteur économique du vêtement lui a permis de gagner la confiance et la collaboration de régulateurs économiques sans lesquels la pérennité et la stabilité du marché de factures de complaisance ne sauraient s'expliquer. L'alliance tripartite formée entre Leonard Cohen, la Banque Toronto Dominion et le vérificateur comptable externe, Bernard Stotland, 
constitue une caractéristique importante, voire centrale, de cette fraude fiscale.

Cosson (1971) a déjà souligné, dans son importante étude consacrée aux «industriels de la fraude fiscale», le rôle des banques dans la mise sur pied des marchés de factures de complaisance. La relation entre les banques et les animateurs de fraudes fiscales dépeinte par Cosson relève davantage de l'assistance passive qu'active, car l'institution financière ferme les yeux sur les entrées et les sorties de fonds apparaissant suspects sous les angles des montants et de la chronologie. La prévalence de cette forme de collaboration peut s'expliquer par les caractéristiques des fraudeurs et des fraudes fiscales rapportées. En effet, Cosson (1971), comme De Brie et Charpentier (1975), font ressortir l'implication d'individus qui ne sont pas intégrés dans les industries au sein desquelles ils opèrent. Les auteurs citent notamment la participation de «taxis», soit des prête-noms recrutés parmi les délinquants ordinaires, ainsi que le recours aux compagnies de façade comme caractéristiques communes à ces réseaux de facturation fictive. Leur structure éphémère explique l'intérêt moindre des banques à participer à un stratagème délictueux, puisque le client corporatif représente davantage de risques que de gains potentiels, faute de structure corporative légale et profitable.

Le cas de figure révélé dans l'affaire «Ventex» est très différent. Plusieurs renseignements tirés des transcriptions judiciaires et des preuves présentées au procès tendent à corroborer deux hypothèses: d'une part, l'assistance des employés de la Banque Toronto Dominion aux pratiques illégales de Cohen et, d'autre part, la tolérance du vérificateur comptable, Bernard Stotland, pour des anomalies comptables. Bien qu'elles n'aient pas pour tâche de détecter la fraude, les deux parties ont ignoré maints éléments d'information qui imposaient d'entreprendre des vérifications approfondies des activités commerciales de Shildan ou de se dissocier de ses activités.

Deux principaux aspects des affaires menées par Shildan, dont ont eu connaissance à la fois certains employés de la Banque Toronto Dominion, par leur position stratégique, et Bernard Stotland, par ses obligations de vérification, sont les signes d'un risque manifeste. Le premier est constitué de deux éléments: d'une part, l'expansion hors du commun des ventes et des bénéfices de la compagnie, à partir du moment où son compte a été transféré à la Banque Toronto Dominion, et, d'autre part, le volume de ses ventes en comparaison avec sa capacité de production, en termes de machinerie, d'heures de sous-traitance 
payées et d'achats de stocks de marchandises. Les sommes extraordinaires payées comptant aux entreprises de façade constituent le second indice $^{14}$.

Les informations recueillies dans les transcriptions judiciaires et dans les preuves des procès tracent le portrait d'une institution financière qui ferme les yeux sur des augmentations extraordinaires de ventes et de bénéfices, malgré l'inquiétude exprimée par les dirigeants de la banque à propos de la légalité des transactions de Shildan. Malgré la mise au jour d'indices flagrants de fraude et les pressions du vérificateur externe imposé par la banque, un cadre est également prêt à empêcher la tenue de vérification comptable pendant plus de trois mois. Deux des principaux fournisseurs de Shildan, Debco et Riegel, les compagnies de façade de Cohen avec lesquelles la société transige en comptant des montants atteignant plus d'un million de dollars mensuellement, ne sont connues ni de la banque ni du vérificateur externe. Enfin, ce dernier ne semble pas s'intéresser au fait que la compagnie n'ait pas, en termes de machinerie, les moyens de production qui justifient les ventes rapportées. Les heures de sous-traitance ne sont pas examinées et la vérification des stocks de marchandises n'est réalisée qu'en partie, ce qui est une pratique non conforme aux normes.

Pris isolément, la plupart des indices examinés dans l'analyse ne constituent pas à eux seuls une démonstration convaincante de la complaisance de la banque et de l'aveuglement volontaire du vérificateur externe. Additionnés, ils soulèvent toutefois un doute important sur le rôle prépondérant de ces deux acteurs, particulièrement le premier, dans la mécanique bien lubrifiée de cette fraude fiscale. L'alliance de Cohen avec certains employés de la succursale Saint-Laurent/PrinceArthur était nécessaire à l'expansion de son commerce de tissus. Ses activités d'accommodation constituaient en quelque sorte la garantie financière à l'obtention de crédit. Il fallait donc à Cohen une banque où quelques employés seraient disposés à fermer les yeux sur des signes explicites de fraude, ce qui équivaut, en retenant l'hypothèse conservatrice, à une complaisance passive. L'histoire révèle cependant qu'ils ont été utiles pour leur client, de façon proactive. Stotland, pour sa part, était un bon allié dans la mesure où, à la lumière des informations colligées dans les transcriptions judiciaires, il a accepté de jouer le jeu

14. Le paiement «comptant» se fait sans emploi de crédit, principalement par chèque ou en argent liquide. 
de la naïveté et de ne pas chercher à approfondir ses vérifications comptables tant que les indices n'ont pas été trop explicites. La patience de Stotland pourrait fort bien s'expliquer par son désir de maintenir la lucrative collaboration qu'il entretenait avec la succursale bancaire, à qui il rendait des services gratuitement en échange de la promotion de sa firme comptable auprès de ses clients.

\section{L'anomalie apparente de l'avalanche des procédures pénales dans l'affaire "Ventex »}

Il est généralement admis que les délinquants d'affaires sont rarement l'objet de poursuites pénales. Shapiro $(1985: 210)$ montre, dans son étude sur le traitement judiciaire des auteurs de fraudes liées aux valeurs mobilières, que moins du quart $(21 \%)$ des délinquants d'affaires au statut élevé, impliqués dans des fraudes liées aux valeurs mobilières jugées sérieuses, sont renvoyés devant les tribunaux criminels et que $8 \%$ seulement de toutes ces plaintes se soldent par une sentence d'emprisonnement. Les tribunaux criminels apparaissent ainsi comme un recours passablement exceptionnel. En ce qui concerne les organes administratifs français compétents en matière de délits fiscaux, Lascoumes (1983: 137) explique qu'ils perçoivent le recours à l'appareil pénal «[...] comme étant d'abord l'échec de leur propre action régulatrice». Une thèse centrale de Shapiro (1985) est que les organismes de contrôle préféreront les poursuites civiles ou administratives, lorsqu'elles sont disponibles, aux solutions extrêmes que sont soit l'inaction soit les poursuites criminelles. Trois facteurs expliqueraient le peu de motivation des organes de contrôle à saisir les tribunaux pénaux. Premièrement, les règles de preuves sont plus strictes et les enquêtes doivent être plus rigoureuses. Étant donné les ressources limitées à leur disposition, ils préferent diviser leur temps entre un plus grand nombre de cas. Deuxièmement, en référant une affaire au système pénal, les procureurs des organismes de contrôle des valeurs mobilières perdent le contrôle de la cause au profit du procureur de la couronne, dont ils sont généralement insatisfaits de la compétence à mener à bien une enquête criminelle et à obtenir une condamnation. Shapiro note que les facultés réparatrices des sanctions pénales sont peu attrayantes puisqu'elles incluent rarement la restitution des sommes fraudées. En outre, quand le tribunal considère que des pénalités financières seraient excessives, la peine infligée est 
perçue par ceux qui ont initié les poursuites comme inapte à prévenir la récidive.

\section{Chronologie des poursuites civiles et pénales} dans l'affaire "Ventex»

En juin 1976, un article paru dans le quotidien The Gazette identifie la Banque Toronto Dominion comme une victime du marché de factures de complaisance. Le journaliste rapporte que la banque a été lésée de près de deux millions de dollars par de fausses listes de comptes à recevoir. Elle apparaît ainsi comme la victime dénonciatrice de cette fraude fiscale. À partir de juin 1976, elle est l'instigatrice non seulement de la requête en faillite de Shildan, mais de trois poursuites civiles distinctes et de deux poursuites criminelles. La dernière poursuite civile n'a été abandonnée qu'en 1996, soit vingt ans après le début cette «saga» judiciaire.

L'examen approfondi des démarches judiciaires entreprises contre les divers acteurs impliqués dans cette affaire génère des informations éloquentes sur le traitement judiciaire d'une fraude fiscale complexe. Il doit inclure l'étude de la nature des poursuites (criminelles, civiles ou administratives) ainsi que des motivations avouées ou implicites des parties sur lesquelles l'analyse de l'ordre dans lequel elles ont été entreprises jette un éclairage utile.

Dès la mise au jour de l'affaire, la Banque Toronto Dominion entreprend des démarches dont l'objectif évident est de récupérer le crédit de deux millions de dollars accordé à Shildan Knitting Mills Limited, dont est garant Leonard Cohen. Elle dépose en juin 1976 une requête en faillite contre Shildan. Étant donné que la banque ne récupère qu'environ $600000 \$$ sur les garanties qu'elle possède, elle entreprend en mars 1977 une poursuite civile contre Cohen, afin de récupérer le solde de sa créance. Cette démarche est un succès apparent, puisqu'elle obtient contre lui un jugement de 1288801 \$, plus les intérêts et les dépens. Toutefois, une lettre des avocats de l'institution financière démontre que, dans les faits, seule la somme de 3302 \$ est perçue par les syndics de faillite et remise à la Banque Toronto Dominion ${ }^{15}$.

La banque cherche à recouvrer le solde de la perte auprès de sa compagnie d'assurance qui refuse la réclamation. Elle intente donc contre elle, en octobre 1977, une poursuite civile. En outre, elle entame en

15. B.T.D. c. Cohen, jugement [13/10/77]. 
novembre 1977 une poursuite contre la firme de vérificateurs externes, dont fait partie Stotland, qu'elle accuse d'avoir été négligente dans l'exécution de ses mandats de vérification et qu'elle tient pour responsable de la perte de 1,3 million de dollars. La firme s'en défend et soutient que la Banque Toronto Dominion commet une erreur en droit en la poursuivant alors qu'elle n'a pas épuisé tous les autres recours de recouvrement. Elle mentionne entre autres qu'un jugement doit préalablement être prononcé dans la poursuite contre la compagnie d'assurance. Cette poursuite est donc également laissée en plan, temporairement.

Les poursuites intentées dès le mois de février 1978 contre Leonard Cohen et Harry Hartman le sont devant les instances criminelles. Elles ne peuvent viser la réparation, puisque les démarches les plus efficaces à cet égard se sont soldées par un échec ou sont en attente (compagnie d'assurance et vérificateurs externes). La mise en plan de la poursuite civile contre la compagnie d'assurance n'est pas officiellement justifiée et laisse sous-entendre que la banque doit d'abord procéder à d'autres démarches. Il est important de noter que, contre toute attente, aucune démarche judiciaire n'a été entreprise contre Cohen par le ministère du Revenu. Le fisc a choisi de poursuivre les clients accommodés plutôt que l'accommodateur lui-même. Cela tient en partie au fait que Leonard Cohen déclarait indirectement au fisc ses ventes fictives et qu'il avait «collaboré» avec les enquêteurs du fisc à la suite du dévoilement de l'affaire et en partie au fait que la Banque entreprenait les coûteuses poursuites. La compréhension de l'implication de la banque dans les tribunaux criminels nécessite l'analyse de l'ensemble des poursuites intentées.

La banque semble n'avoir rien laissé au hasard pour assurer le succès de la démarche devant les tribunaux criminels, puisque le plaidoyer de culpabilité de Harry Hartman et la tenue de procès séparés expliqueraient, en échange d'une sentence réduite, l'obtention de son témoignage dans le procès de Leonard Cohen. Une entente similaire visant à recueillir son témoignage contre Cohen pourrait également rendre compte de l'absence de poursuite criminelle contre le comptable Sam Karpman à titre de co-conspirateur, qu'avait pourtant initialement envisagée la banque $^{16}$. La grâce «accordée» à Karpman est autrement surprenante, étant donné son rôle central dans l'affaire. Il a d'ailleurs été sévèrement

16. Sur l'importance de la négociation de plaidoyer ("plea bargaining») en matière de délinquance d'affaires, voir Hagan, Nagel et Albonetti (1980). 
puni par le tribunal des professions de l'Ordre des comptables agréés qui l'a radié de façon permanente du Tableau de l'ordre, en juin 1979.

Leonard Cohen et Harry Hartman sont accusés d'avoir obtenu des facilités de crédit sous de fausses représentations et d'avoir comploté dans ce but. Cohen, qui plaide non coupable, est jugé coupable en décembre 1981. Sa sentence initiale de trois ans de pénitencier est réduite, en appel, à un an de prison. Hartman, qui plaide coupable aux accusations qui pèsent contre lui, est condamné, en janvier 1983, à une sentence suspendue de six mois d'emprisonnement en plus de devoir payer une amende de $50000 \$$, à défaut de quoi il devra purger six mois de prison.

En février 1981, la banque reprend la poursuite civile intentée contre la compagnie d'assurance en 1977. La compagnie d'assurance allègue que les prêts ne sont pas couverts par le contrat. La Banque argue pour sa part qu'un crédit n'est pas un prêt et qu'elle peut se prévaloir d'une autre clause qui couvre les pertes encourues par l'assuré faisant affaires de bonne foi, au cours de transactions basées sur des documents «[...] counterfeited or cancelled or forged or raised or otherwise altered or lost or stolen, $[\ldots] »^{17}$. Le demandeur est débouté en première instance au mois de décembre 1981. D'une part, le juge confirme que la perte en cause est visée par la clause d'exclusion des prêts et il émet l'opinion que la banque a été négligente dans ses transactions avec Coben, donc qu'elle ne respecte pas la condition de la bonne foi dont il est fait mention dans la clause à laquelle elle fait référence ${ }^{18}$. La Banque Toronto Dominion porte la cause en appel. Elle est de nouveau déboutée en septembre 1994. Les juges de cette cour statuent que le lien entre les notions de négligence et de mauvaise foi leur semble questionnable mais ils confirment qu'un crédit est une forme de prêt ${ }^{19}$. La banque n'est toutefois pas blanchie de cette allusion à la négligence.

Le recours contre la compagnie d'assurance ayant échoué, la Banque Toronto Dominion reprend la poursuite contre la firme de vérificateurs comptables, en juillet 1996, date à laquelle un désistement est signé par les deux parties qui se partagent les frais des procédures. Aucune justification n'est donnée pour expliquer ce désistement. Fait étrange, contrairement à la pratique courante pour des assurés ${ }^{20}$, la firme de

17. B.T.D. c. C.I.C., jugement en première instance [22/12/81], p.19.

18. Ibid.

19. B.T.D. c. C.I.C., jugement en appel [08/09/94].

20. B.T.D. c. C.I.C., preuves: correspondance interne [25/06/76]. 
vérificateurs n'est pas représentée par ses assureurs lors des comparutions en cour. La seule explication possible est que les assureurs de la firme comptable ont également refusé de couvrir la perte, parce qu'ils ont statué que les comptables avaient été parties prenantes à la fraude ${ }^{21}$.

Compte tenu de la rareté des poursuites pénales à l'endroit des délinquants d'affaires, la poursuite criminelle intentée contre le maître d'œuvre du marché de factures de complaisance analysé par la Banque Toronto Dominion semble de prime abord atypique. Comment rendre compte de cette particularité?

Tout d'abord, la Banque Toronto Dominion savait, en entamant une poursuite criminelle contre Cohen, que ses chances de récupérer les fonds perdus étaient infimes. Une fois les démarches judiciaires civiles et administratives terminées, on aurait donc pu s'attendre à ce que l'institution financière laisse le ministère du Revenu procéder. De toute évidence, la Banque Toronto Dominion poursuivait d'autres objectifs que ceux de restitution et de répression, puisque les coûts engendrés par les poursuites subséquentes étaient à eux seuls dissuasifs. L'étude des rapports de collusion entre certains employés de la Toronto Dominion et Leonard Cohen montre que cette banque était à la fois victime et partie prenante au stratagème des factures de complaisance. Les allusions publiques à la négligence de la banque dans la poursuite intentée contre sa compagnie d'assurance imposaient une réponse à la hauteur. On peut supposer que la Toronto Dominion avait à cœur de «protéger sa réputation" auprès des autres banques et de sa clientèle. Il est fort possible aussi qu'elle ait voulu signaler, haut et fort, son intolérance à l'endroit de ce type d'écarts de conduite de ses propres employés et de ses associés professionnels (en l'occurrence, les vérificateurs comptables). La poursuite civile intentée contre le vérificateur comptable impliqué dans l'affaire «Ventex» en est un indice probant malgré son désistement expliqué notamment par l'existence de chances minimales d'obtenir un gain pécuniaire, étant donné l'absence de couverture d'assurance professionnelle suggérée par les documents à notre disposition, mais aussi le risque de dévoilement d'autres ratés de la part des employés de la banque, de concert avec le vérificateur.

21. Vérification faite, il n'existe aucune enquête disciplinaire ou sanction dans le dossier de Stotland à l'Ordre des comptables agréés, ni à cette époque ni plus tard. 


\section{Conclusion}

La plupart des recherches sur les délits corporatifs s'intéressent soit à la mécanique du délit, soit à son traitement judiciaire. Nous avons trouvé plus utile d'intégrer les deux dimensions. Pour comprendre la mécanique de cette fraude fiscale, il a fallu identifier les intérêts précis des protagonistes impliqués. Ces intérêts expliquent notamment les particularités des poursuites judiciaires dont elle a fait l'objet. En revanche, ce sont ces poursuites judiciaires, surtout civiles d'ailleurs, qui permettent de mieux saisir non seulement les intérêts spécifiques des protagonistes de la fraude mais également le «succès» et la longévité de celle-ci.

Il est également important de souligner que la délinquance d'affaires mobilise d'autres passions que l'appât du gain et la maximisation des bénéfices illicites. Le patron de Ventex a fait office, pendant de longues années, de vendeur officiel de factures de complaisance dans le milieu de l'industrie montréalaise du vêtement pour dames. Toutefois, ce délinquant d'affaires déclarait au fisc les bénéfices qu'il retirait de ses ventes fictives et demeurait un entrepreneur bien intégré dans le milieu du textile. L'effondrement de ce marché de factures de complaisance n'est pas imputable à ses failles internes mais à l'incapacité de l'accommodateur de développer ce marché illicite dans un contexte de récession qui avait fait chuter son chiffre légal d'affaires. Les poursuites intentées par la Banque Toronto Dominion ne relèvent pas d'une logique comptable, mais d'une poursuite d'honneur visant à restaurer sa réputation d'intégrité institutionnelle par voie de règlement de compte à l'endroit du vérificateur externe et de certains de ses propres employés. Cette poursuite a donné les résultats escomptés: emprisonnement de Leonard Cohen, radiation civile du vérificateur externe, mise à la retraite anticipée d'employés de la banque, repentir rapide et dénonciations massives d'un grand nombre d'acheteurs de factures de complaisance.

Lors d'une rencontre organisée à l'automne 1999, des fonctionnaires affectés au Bureau de la lutte contre l'évasion fiscale du ministère du Revenu du Québec ${ }^{22}$ nous ont décrit les formes contemporaines de fraude fiscale de factures de complaisance pratiquées dans l'industrie du vêtement. Il semblerait que la mécanique de ces factures de complaisance differe en tous points de celle qui caractérisait l'affaire «Ventex»

22. Communication personnelle de messieurs Serge Lortie et Gilles Bernard, dont la collaboration a été fort utile. Nous les en remercions. 
dans les années 1960 et 1970. Les accommodateurs seraient des entreprises fantômes : absence totale de locaux d'affaires, d'infrastructure de production, de véhicules, de dépenses d'exploitation; une luxuriance de prête-noms endossés par des personnes inexistantes ou par des acteurs qui ne sont nullement intégrés au secteur d'activité des compagnies accommodées; une espérance de vie éphémère des compagnies accommodatrices. Un portrait fort similaire à celui que dressaient Jean Cosson (1971) et De Brie et Charpentier (1975) dans leurs travaux sur les fraudes fiscales des industriels français à la suite de la mise en place de la TVA dans les années 1970. Il est possible que l'introduction au Canada et au Québec des taxations similaires de la TPS et de la TVQ soit responsable de cette «réorganisation» des pratiques de fraude fiscale dans le milieu des affaires.

La mise au jour récente de fraudes liées à la TPS qui sévissent dans l'industrie automobile suggère toutefois la survie de fraudes durables et nécessite la réalisation d'une analyse comparative entre ces activités illégales et les pratiques frauduleuses «intégrées» mises sur pied par Leonard Cohen dans les années 1960. Le principe de base de ces fraudes fiscales réside dans l'achat et la vente fictive de véhicules automobiles dont on réclame au fisc après coup la taxe sur les produits et les services payée à l'achat. Ces fraude ont d'emblée, comme caractéristique commune, la difficulté pour le fisc et les corps policiers de prouver le crime, étant donné qu'il est fort difficile, lorsqu'il s'agit de véritables entreprises et sans délateurs particulièrement informés, de distinguer les vraies des fausses transactions. Cette propriété explique, du moins en partie, la méconnaissance des autorités à l'égard des nouvelles formes que prennent les illégalismes de longévité remarquable.

\section{Références}

Akerman, S.-R. (1978). Corruption: a study in political economy. New York: Academic Press.

Alm, J. (1996). Explaining tax compliance. In S. Pozo (ed.), Exploring underground economy: studies of illegal and unreported activity (103-127). Kalamazoo, Michigan: W.E. Upjohn Institute for Employment Research.

Baker, W., \& Faulkner, R. (1993). The social organization of conspiracy: illegal networks in the heavy electrical equipment industry. American Sociological Review.

Barnett, H. (1984). Tax evasion by proxy: the gray market in welfare capitalism. Contemporary Crises, 8, 107-123. 
Bernard, M., Lauzon, L.-P., \& Poirier, M. (1995). La désinvolture des gouvernements face à l'évitement des impôts par les compagnies. Université du Québec à Montréal: Département des sciences comptables.

Clinard, M. (1979). Illegal corporate behavior. U.S. Dept. of Justice, Law Enforcement Assistance Administration, National Institute of Law Enforcement and Criminal Justice.

Clinard, M. (1990). Corporate corruption: the abuse of power. New York: Preager. Clinard, M., \& Yeager, P. (1980). Corporate Crime. New York: The Free Press.

Cosson, J. (1971). Les industriels de la fraude fiscale. Paris : Éditions du Seuil.

Cowell, F.A. (1991). Cheating the governement: the economics of evasion. Cambridge, MA : MIT Press.

DeBrie, C., \& Charpentier, P. (1975). Dossier F .. comme dans fraude fiscale. Paris : Éditions Alain Moreau.

Denzin, N. (1977). Notes on criminogenic hypothesis: a case study of the american liquor industry. American Sociological Review, 42 (décembre), 905920.

Elffers, H. (1991). Income tax evasion: theory and measurement. Erasmus Centre for Sociolegal Tax Research, Deventer: Kluwer.

Faberman, H. (1975). A criminogenic market structure: the automobile industry. The Sociological Quaterly, 16 (automne), 438-457.

Friedrichs, D. (1996). Trusted criminals: white-collar crime in contemporary society. Belmont: Wadsworth Publishing Company.

Gray, A.D. (1976). Income tax evasion scheme involves nearly 200 firms. The Gazette, le 8 juin 1976.

Gray, A.D. (1977). Manufacturers' plea for leniency refused. The Gazette, le 29 juillet 1977.

Hagan, J., Nagel, I., \& Albonetti, C. (1980). The differential sentencing of white-collar offenders in ten federal district courts. American Sociological Review, 45 (octobre), 802-820.

Klepper, S., \& Nagin, D. (1989). The deterrent effect of perceived certainty and severity of punishment revisited. Criminology, 27 (4), 721-740.

Lascoumes, P. (1983). Sanction des fautes ou gestion des illégalismes: l'hétérogénéisation du droit pénal. Un exemple, la répression fiscale. Revue interdisciplinaire d'études juridiques, 10, 125-156.

Leonard, W., \& Weber, M. (1970). Automakers and dealers: a study of criminogenic market forces. Law and Society Review, 4 (3), 407-424.

Levi, M. (1987). Regulating fraud: white-collar crime and the Criminal Process. London: Tavistock Publications.

Marsden, W. (1982). How they worked the great garment trade tax evasion scam. The Gazette, le 30 janvier 1982.

Nagin, D., \& Klepper, S. (1987). The anatomy of tax evasion. Carnegie Mellon University.

Pyle, D. (1989). Tax evasion and the black economy. London: Macmillan. 
Reuter, P. (1993). The cartage industry in New York. In M. Tonry \& A. Reiss (eds), Beyond the law: crime in complex organizations (149-199). Crime and Justice: A Review of Research, 18. Chicago: University of Chicago Press.

Shapiro, S. (1985). The road not taken : the elusive path to criminal prosecution for white-collar offenders. Law and Society Review, 19 (2), 179-217.

Shover, N., \& Bryant, K.M. (1993). Theoritical explanations of corporate crime. In M. Blankenship (ed.), Understanding corporate criminality (141-176). New York: Garland Publishing, Inc.

Stalans, L., Smith, K., \& Kinsey, K. (1987). Intentions to evade taxes: interpersonal communication, audit experience and opportunity. Août 1987, American Bar Foundation: document inédit.

Thurman, Q. (1989). General prevention of tax evasion: a factorial survey approach. Journal of Quantitative Criminology, 5 (2), 112-134.

Tonry, M., \& Reiss, A. (1993). Organizational crime. In M. Tonry \& A. Reiss (eds), Beyond the law : crime in complex organizations (1-10). Crime and Justice: A Review of Research, 18. Chicago: University of Chicago Press.

Tremblay, P., Cusson, M., \& Morselli, C. (1998). Market offenses and limits to growth. Crime, Law and Social Change, 29, 311-330.

Varma K., \& Doob, A. (1998). Deterring economic crimes: the case of tax evasion. Revue canadienne de criminologie, 40 (2), 165-184.

Wiegand, B. (1994). Black money in Belize: the ethnicity and social structure of black-market crime. Social Forces, 73 (1), 135-154.

Yin, R. (1989). Case study research: design and methods. Applied Social Research Methods Series, 5. Newbury Park: Sage Publications Inc.

\section{ANNEXE 1}

\section{Abréviations - terminologie}

$\begin{array}{ll}\text { B.T.D. } & \text { Banque Toronto Dominion } \\ \text { C.I.C. } & \text { The Continental Insurance Company } \\ \text { C.A. } & \text { Cour d'appel } \\ \text { C.S. } & \text { Cour supérieure } \\ \text { C.S.P. } & \text { Cour des sessions de la paix } \\ \text { D.F. } & \text { Division des faillites } \\ \text { jug. } & \text { Jugement } \\ \text { Shildan } & \text { Shildan Knitting Mills Limited } \\ \text { t. } & \text { Témoignage } \\ \text { t.j. } & \text { Transcription judiciaire }\end{array}$

\section{Abréviations - causes}

1- Sa Majestée la Reine c. Leonard Cohen, C.S.P. [01-001234-787] Leonard Cohen c. Sa Majestée la Reine, C.A. [10-000046-829]

Abréviation: R. c. Cohen

Abréviation: Cohen c. $R$. 
2- Sa Majestée la Reine c. Harry Hartman, C.S.P. [01-001235-784] Abréviation: R. C. Hartman

3- Banque Toronto Dominion c. The Continental Insurance Company, C.S. [05-022113-771]

Banque Toronto Dominion c. The Continental Insurance Company, C.A. Abréviation: B.T.D. C. C.I.C.

4- Banque Toronto Dominion c. Leonard Cohen, C.S. [05-005144-777] Abréviation: B.T.D. c. Cohen

5- Banque Toronto Dominion c. Bernard Stotland, Mark B. Wasserman, Nathan Bratt et Louis Grossbaum, C.S. [05-024412-775]

Abréviation: B.T.D. c. Stotland

6- Division des faillites c. Shildan Knitting Mills Limited [11-000809-76]

Abréviation: D.F. c. Shildan

7- Samuel Karpman c. Pierre Fortier, C.P. (Tribunal des professions) [02-027326-797]

\section{Abréviations - références \\ (témoignages, éléments de preuves et jugements)}

Cause, t.j. (date du témoignage), t. de (nom du témoin), p. (de l'extrait cité). Cause, preuves: (élément de preuve) (date - s'il y a lieu).

Cause, jug. (date du jugement), p. (de l'extrait cité). 